\title{
Density of nucleation centres of the normal state and magnetic interactions in superheated superconducting granules
}

\author{
D. Hueber $\left({ }^{*}\right)$, C. Valette $\left({ }^{*}\right)$ and G. Waysand $\left({ }^{*}\right)$ \\ Laboratoire de Physique des Solides, Université Paris-Sud, Bât. 510, 91405 Orsay, France
}

(Reçu le 13 août 1980, réviséle 16 octobre, acceptéle 22 octobre 1980)

\begin{abstract}
Résumé. - Par la méthode de détection d'impulsion dans l'observation de la rupture de surchauffe de grains supraconducteurs, on mesure le pourcentage du nombre de grains en état de surchauffe en fonction de leur taille dans un champ magnétique appliqué donné. Deux extrapolations différentes déterminent : 1) la densité des centres de nucléation de l'état normal, 2) le pourcentage de grains surchauffés que l'on aurait s'ils étaient sans défaut. La courbe de surchauffe ne prenant en compte que les interactions diamagnétiques est tracée.
\end{abstract}

\begin{abstract}
The pulse-detection method for the observation of the transition to the normal state of superconducting superheated granules measures the percentage of the number of superheated granules as a function of their size for a given applied magnetic field. Two different extrapolations provide : 1) the density of nucleation centres of the normal state and 2) the percentage of superheated granules that would be found if they were defect free. The superheated branch of the hysteresis cycle involving only diamagnetic interactions is drawn.
\end{abstract}

A suspension of small spherical granules of type I superconductors has at $T<T_{\mathrm{c}}$, a non reversible transition in a magnetic field. The usual method of observation is by measurement of the variation of the inductance of a coil surrounding the granules. For a single granule, one gets a perfectly rectangular cycle [1,2], and extensive comparison with theories of the superheated field $H_{\text {sh }}$ have been made [1]. For a collection of granules, continuous variations of the inductance are observed. The shape of the superheated branch of the hysteresis cycle varies from sample to sample; this has been attributed to the presence of nucleation centres of the normal state which are responsible for flipping of the granules to the normal state at $H_{\mathrm{c}}<H<H_{\mathrm{sh}}\left(H_{\mathrm{c}}\right.$ : thermodynamical critical field). However, it is obvious that with the usual filling factors (about $10 \%$ of the volume within the coil), the local field around the granules is different from the applied field due to diamagnetic interactions between granules. These interactions contribute to the broadening of the superheated branch, but because the inductance method is integrative, it cannot distinguish between these magnetic interactions and the presence of nucleation centres; both effects contribute to the width of the superheated branch of the hysteresis cycle.

(*) Present address : Groupe de Physique des Solides de l'E.N.S., Université Paris VII, Tour 23, 2, place Jussieu, 75221 Paris Cedex 05, France.
The aim of this paper is to demonstrate that the pulse method [2] can fulfil this task : the contribution of defects can be experimentally evaluated, and the remaining width of the transition is thus due only to the diamagnetic interactions between the granules. In this case, the coil is a pick-up coil connected through a transmission line to the input of a special charge preamplifier. This system works as a fluxmeter operating in the microsecond range. Each granule provides a pulse proportional to its volume. The pulses are sent to a counting scale and in such a method, the hysteresis cycle gives directly the number of grains which have already flipped to the normal state for a given applied magnetic field. This method, compared to the inductance method has an additional adjustable physical parameter : the threshold of the counting scale. The variation of this threshold provides an in situ size selection of granules. Therefore, for the same sample, it is possible to obtain the hysteresis cycle of all the granules of a given range of size.

We have realized such an experiment with a foil of paraffin $81 \times 81 \times 0.300 \mathrm{~mm}$ containing $10 \%$ by volume of tin granules with diameters between 12 and $33 \mu$ at $1.7 \mathrm{~K}$, with the magnetic field perpendicular to the foil. The dispersion of the granules in the paraffin is far from homogeneous and we estimate by observation from place to place of the optical transparency of the foil that there are clusters of a fraction of millimeter in size in which the filling factor is increased to as much as $40 \%$. The pick-up coil is 
flat and U-shaped, and of the type described in [3] : $81 \mathrm{~mm}$ length, $0.3 \mathrm{~mm}$ width. It is stuck on the central part of the foil. In this situation, even when one is rather close to $H_{\mathrm{sh}}$, the demagnetization factor for the detected granules is still the demagnetization factor of a flat disk of granules in a perpendicular magnetic field.

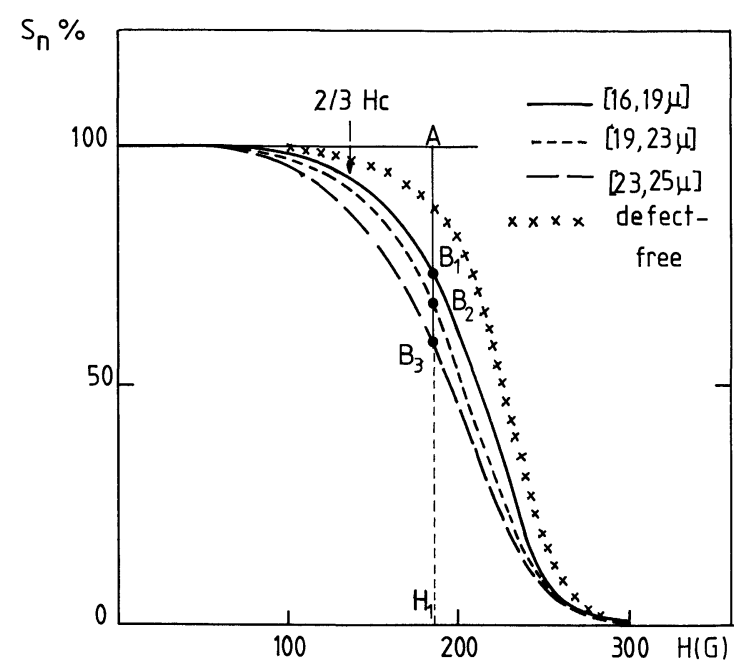

Fig. 1. - Percentage $S_{\mathrm{n}} \%$ of the number of superheated grains in the sample as a function of the applied magnetic field, for three different electronic thresholds (corresponding range of size $16<D<19 \mu, 19<D<23 \mu, 23<D<25 \mu, D$ : diameter of grains). Extrapolated curve for defect-free granules (involving only diamagnetic interactions between grains).

The results are shown in figure 1 where the percentage, $S_{\mathrm{n}} \%$, of superheated grains in the sample is recorded as a function of the applied magnetic field for three different electronic thresholds. The intersection of the vertical line $H=H_{1}$ with the various curves, $B_{1} B_{2} B_{3}$, gives the percentage $\left(A_{1}, A B_{2}\right.$, $\left.\mathrm{AB}_{3}\right) N_{\mathrm{n}} \%=100-S_{\mathrm{n}} \%$ of granules of various size that have left the superheated state. $B_{1}, B_{2}, B_{3}$ are at constant magnetic interactions because the magnetic field is constant and all the superconducting granules are present at fixed sites in the sample no matter what the counting threshold is. The higher is the threshold (the bigger are the measured granules), the larger is $N_{\mathrm{n}} \%$. From that, we deduce that the variation of $N_{\mathrm{n}} \%$ with the threshold at a given applied magnetic field is due only to the existence of nucleation centres. Since all the granules are produced in the same ultra-sonorization of melted tin mixed in tension active oil, it is worthwhile to check the hypothesis that the nucleation centres (which are surface defects of the granule) have a density independent of the size. For this purpose, we have plotted in figure 2, $N_{\mathrm{n}} \%$ as a function of $D^{2}(D=$ diameter $)$ for various applied fields; $D^{2}$ is proportional to the external surface of the selected granules. We get a perfectly linear function which can be represented by

$$
N_{\mathrm{n}}=N_{\mathrm{n} 0}+\beta(H) D^{2}
$$

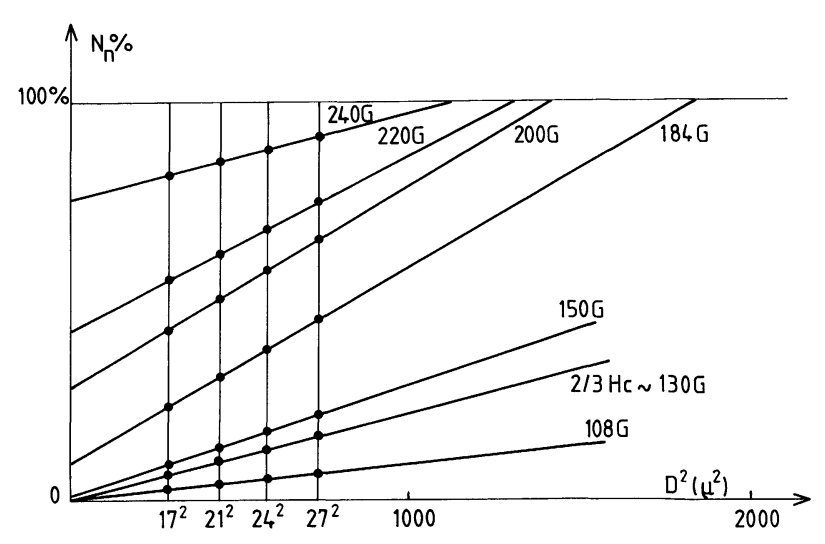

Fig. 2. - Percentage $N_{\mathrm{n}} \%$ of the number of grains that have left the superheated state, for different values of the applied magnetic field, as a function of $D^{2}$.

where $\beta(H)$ is constant for a given magnetic field, representing the increase in $N_{\mathrm{n}}$ for an increment of $\pi / 4$ unit in the surface of the granules. This result means that the number of nucleation centres on a granule is proportional to its surface area. The product $\beta(H) D^{2}$ represents the contribution of the surface defects. $N_{\text {no }}$ is obtained from the intersection of the vertical axis $D=0$ with the line given by equation (1). $N_{\text {no }}$ is thus the percentage of the number of granules which would have flipped even if the granules did not contain nucleation centres : $N_{\mathrm{n} 0}$ is the defectfree value of $N_{\mathrm{n}} \%$ at $H$. By inferring $N_{\mathrm{n} 0}$ for each field in figure 1 , we obtain the defect-free superheated branch of the hysteresis cycle. This is the ideal cycle for the same collection of granules with the same distribution of sites. In this way, the contribution of nucleation centres is completely separated from that due to magnetic interactions without any adjustable parameters. In figure 2, the intersection of each line for a given $H$ with the line $N_{\mathrm{n}} \%=100 \%$ also provides significant information : at this point, all the granules of the collection for a given field will have flipped, thus the abscissa of this point determines the maximum radius for a granule to remain superconducting at the corresponding magnetic field. For the line $H=200 \mathrm{G}$, we get $D^{2}=1420 \mu^{2}$, $D=38 \mu$. This value is in qualitative agreement with our own observations on numerous samples : for tin it is very difficult to observe superheating in such big granules.

In summary, we are able to separate the contribution of nucleation centres from magnetic interactions in the superheated branch of the hysteresis cycle of a collection of superconducting granules. Since the presence of nucleation centres lowers the energy threshold for flipping of the granules, we will use the result of this work for the evaluation of the energy threshold of the granules under ionizing irradiation, in future studies. 


\section{References}

[1] Feder, J. and Mac Lachlan, D. S., Phys. Rev. 177 (2) (1969) 763.

[2] Valette, C. and Waysand, G., Dynamic study of the transition of a single superheated superconducting granule to the normal state. Proceedings of LT 14. Helsinki 2 (1975) 183.
[3] Behar, J., Cardi, J. M., Herszberg, B., Hueber, D., Valette, C. and WAYSAND, G., The development of a superconducting gamma camera, J. Physique Colloq. 39 (1978) C 6-1201. 